\title{
ON A THEORY OF THERMOELASTIC MATERIALS WITH A DOUBLE POROSITY STRUCTURE
}

\section{Ieşan}

Department of Mathematics, "Al.I.Cuza "University and Octav Mayer Institute of Mathematics (Romanian Academy), 700508 Iasi, Romania, iesan@uaic.ro

\section{R. Quintanilla}

Department of Applied Mathematics 2, Polytechnic University of Catalonia 08222, Terrassa, Barcelona, Spain, ramon.quintanilla@upc.edu

\begin{abstract}
In this paper we use the Nunziato-Cowin theory of materials with voids to derive a theory of thermoelastic solids which have a double porosity structure. The new theory is not based on the Darcy's law. In the case of equilibrium, in contrast with the classical theory of elastic materials with double porosity, the porosity structure of the body is influenced by the displacement field. We prove the uniqueness of solutions by means of the logarithmic convexity arguments as well as the instability of solutions whenever the internal energy is not positive definite. Later we use the semigroup arguments to prove the existence of solutions in case that the internal energy is positive. The deformation of an elastic space with a spherical cavity is investigated.
\end{abstract}

Keywords: Porous thermoelastic solids; Existence and uniqueness results; Instability; Unbounded medium with a cavity.

\section{INTRODUCTION}

There has been much written in recent years on the linear theory of elastic materials with double porosity. The origin of this theory goes back to papers of Barenblatt et al. [1,2]. Much of the theoretical progress in the field is discussed in the works [3-13]. The so called double porosity model allows for the body to have a double porous structure: a macro porosity connected to pores in the body and a micro porosity connected to fissures in the skeleton. The materials with double porosity are of interest in geophysics (see, e.g., [2,6,7]) and mechanics of bone [14]. The theory is established with the help of Darcy's law. The basic equations for elastic materials with double porosity involve the displacement vector field, a pressure associated with the pores and a pressure associated with the fissures (see, e.g., $[9,12,13]$ ). We note that in the equilibrium theory the fluid pressures become independent of the displacement vector field. 
In [15], Nunziato and Cowin have established a theory for the behaviour of porous solids in which the skeletal or matrix materials are elastic and the interstices are void of material. The intended applications of this theory are to geological materials such as rocks and soils and to manufactured porous materials such as ceramics and pressed powders. The linear theory of elastic materials with voids has been established by Cowin and Nunziato [15]. The theory of elastic materials with voids has been extensively studied (see, e.g., [16-26] and references therein). In this paper we use the NunziatoCowin theory of materials with voids to derive a theory of thermoelastic solids which have a double porosity structure. The new theory is not based on the Darcy's law. In contrast with the classical theory of elastic materials with double porosity, the porosity structure in the case of equilibrium is influenced by the displacement field.

The plan of this paper is the following. First, we present the non-linear theory of thermoelastic solids with a double porosity structure. Then, the theory is linearized and the basic boundary-initial-value problems are formulated. The logarithmic convexity type argument is used to establish a uniqueness theorem and an instability result. By a semigroup approach we establish an existence result in the dynamical theory. In the final section we consider the equilibrium theory and study the problem of an elastic space with a spherical cavity.

\section{BASIC EQUATIONS}

In what follows we consider a body that at time $t_{0}$ occupies the bounded region $B$ of Euclidean three-dimensional space. The configuration of the body at time $t_{0}$ is taken as the reference configuration. The motion of the body is referred to the reference configuration and a fixed system of rectangular Cartesian axes. We identify a typical particle of the body with its position in the reference configuration. The coordinates of a typical particle in $B$ are $x_{j},(j=1,2,3)$. The coordinate of this particle at time $t$ are denoted by $y_{i}$. We have

$$
y_{i}=y_{i}\left(x_{j}, t\right), \quad\left(x_{j}\right) \in B, t \in I,
$$

where $I=\left(t_{0}, t_{1}\right)$ is a given interval of time. We assume the continuous differentiability of $y_{k}$ with respect to the variables $x_{i}$ and $t$ as many times as required and

$$
\operatorname{det}\left(\frac{\partial y_{i}}{\partial x_{j}}\right)>0 \text { on } B \times I \text {. }
$$

We shall employ the usual summation and differentiation conventions: Latin subscripts (unless otherwise specified) are understood to range over the integers $(1,2,3)$, summation over repeated subscripts is implied and a subscript preceded by a comma denote partial differentiation with respect to the corresponding material coordinate. In what follows, a superposed dot denotes the material derivative with respect to the time.

We consider an arbitrary region $\omega$ of the continuum, bounded by a surface $\partial \omega$ at time $t$, and we suppose that $\Omega$ is the corresponding region at time $t_{0}$, bounded by the surface $\partial \Omega$. Let the outward unit normal at $\partial \Omega$ be $n_{j}$ referred to the rectangular frame 
of reference. We denote by $\nu_{1}$ the volume fraction field corresponding to pores and by $\nu_{2}$ the volume fraction field corresponding to fissures. We postulate the conservation of energy for every regular region $\Omega$ of $B$ and every time, in the form

$$
\begin{aligned}
& \int_{\Omega} \rho_{0}\left(\dot{y}_{i} \ddot{y}_{i}+\kappa_{1} \dot{\nu}_{1} \ddot{\nu}_{1}+\kappa_{2} \dot{\nu}_{2} \ddot{\nu}_{2}\right) d v+\int_{\Omega} \rho_{0} \dot{e} d v= \\
& =\int_{\Omega} \rho_{0}\left(f_{i} \dot{y}_{i}+g \dot{\nu}_{1}+\ell \dot{\nu}_{2}+S\right) d v+\int_{\partial \Omega}\left(T_{i} \dot{y}_{i}+\sigma \dot{\nu}_{1}+\tau \dot{\nu}_{2}+Q\right) d a,
\end{aligned}
$$

where $d v$ and $d a$ are the elements of volume and area in the reference configuration, $\rho_{0}$ is the mass density at time $t_{0}, e$ is the internal energy per unit mass, $\kappa_{1}$ and $\kappa_{2}$ are coefficients of equilibrated inertia, $f_{j}$ is the body force per unit mass, $g$ is the extrinsic equilibrated body force per unit mass associated to macro pores, $\ell$ is the extrinsic equilibrated body force per unit mass associated to fissures, $S$ is the heat supply per unit mass, $T_{i}$ is the stress vector associated with the surface $\partial \omega$, but measured per unit area of the surface $\partial \Omega, \sigma$ is the equilibrated stress corresponding to $\nu_{1}$, associated with the surface $\partial \omega$ but measured per unit area of the surface $\partial \Omega, \tau$ is the equilibrated stress corresponding to $\nu_{2}$, associated with the surface $\partial \omega$ but measured per unit area of the surface $\partial \Omega$, and $Q$ is the heat flux across the surface $\partial \omega$, measured per unit area of $\partial \Omega$. We restrict our attention to the case when $\kappa_{1}$ and $\kappa_{2}$ are given functions on $B$ (see Nunziato and Cowin [15]).

Following the procedure of Green and Rivlin [27], we consider a second motion which differs from the given motion only by a constant superposed rigid translational velocity. We assume that $e, f_{j}, g, \ell, S, T_{j}, \sigma, \tau$ and $Q$ are unaltered by such superposed rigid velocity. If we denote $v_{i}=\dot{y}_{i}$, then the equation (3) is also true when $v_{i}$ is replaced by $v_{i}+a_{i}$, where $a_{i}$ are arbitrary constants, all other terms being unaltered. By subtraction we get

$$
\left[\int_{\Omega} \rho_{0} \ddot{y}_{i} d v-\int_{\Omega} \rho_{0} f_{i} d v-\int_{\partial \Omega} T_{i} d a\right] a_{i}=0,
$$

for all arbitrary constants $a_{j}$. Since the quantities in the square brackets are independent of $a_{j}$, it follows that

$$
\int_{\Omega} \rho_{0} \ddot{y}_{i} d v=\int_{\Omega} \rho_{0} f_{i} d v+\int_{\partial \Omega} T_{i} d a .
$$

From (4), by the usual methods, we obtain

$$
T_{i}=T_{k i} n_{k}
$$

and

$$
T_{k i, k}+\rho_{0} f_{i}=\rho_{0} \ddot{y}_{i} .
$$


Here, $T_{k i}$ is the first Piola-Kirchhoff stress tensor. In view of (5) and (6), the relation (3) reduces to

$$
\begin{aligned}
\int_{\Omega} \rho_{0}\left(\dot{e}+\kappa_{1} \dot{\nu}_{1} \ddot{\nu}_{1}+\kappa_{2} \dot{\nu}_{2} \ddot{\nu}_{2}\right) d v & =\int_{\Omega}\left[T_{k i} v_{i, k}+\rho_{0}\left(g \dot{\nu}_{1}+\ell \dot{\nu}_{2}+S\right)\right] d v+ \\
& +\int_{\partial \Omega}\left(\sigma \dot{\nu}_{1}+\tau \dot{\nu}_{2}+Q\right) d a
\end{aligned}
$$

With an argument similar to that used in obtaining the relations (5), from (7) we get

$$
\left(\sigma-\sigma_{j} n_{j}\right) \dot{\nu}_{1}+\left(\tau-\tau_{j} n_{j}\right) \dot{\nu}_{2}+Q-Q_{j} n_{j}=0 .
$$

where $Q_{j}$ is the heat flux vector, and $\sigma_{j}$ and $\tau_{j}$ are equilibrated stress vectors. If we use (8) in (7) and apply the resulting equation to an arbitrary region, then we find the local form of the conservation of energy

$$
\rho_{0} \dot{e}=T_{k i} v_{i, k}+\sigma_{i} \dot{\nu}_{1, i}+\tau_{i} \dot{\nu}_{2, i}-\xi \dot{\nu}_{1}-\zeta \dot{\nu}_{2}+Q_{j, j}+\rho_{0} S,
$$

where the functions $\xi$ and $\zeta$ satisfy the equations

$$
\sigma_{j, j}+\xi+\rho_{0} g=\kappa_{1} \ddot{\nu}_{1}, \tau_{j, j}+\zeta+\rho_{0} \ell=\kappa_{2} \ddot{\nu}_{2} .
$$

The intrinsic equilibrated body forces $\xi$ and $\zeta$ are defined by constitutive equations. Let us introduce the second Piola-Kirchoff stress tensor $S_{i j}$ by

$$
T_{k i}=y_{i, j} S_{k j} .
$$

The relation (9) can be written in the form

$$
\rho_{0} \dot{e}=S_{k m} y_{i, p} y_{j, k}\left(d_{i j}+\omega_{i j}\right)+\sigma_{i} \dot{\nu}_{1, i}+\tau_{i} \dot{\nu}_{2, i}-\xi \dot{\nu}_{1}-\zeta \dot{\nu}_{2}+Q_{j, j}+\rho_{0} S,
$$

where

$$
2 d_{i j}=\frac{\partial v_{i}}{\partial y_{j}}+\frac{\partial v_{j}}{\partial y_{i}}, 2 \omega_{i j}=\frac{\partial v_{i}}{\partial y_{j}}-\frac{\partial v_{j}}{\partial y_{i}} .
$$

We consider a motion of the continuum which differs from the given motion only by a superposed uniform rigid body angular velocity, and assume that $\rho_{0}, \dot{e}, S_{i j}, \dot{\nu}_{1}, \dot{\nu}_{2}, \sigma_{i}, \tau_{i}, \xi, \zeta$, $Q_{j}$ and $S$ are unlatered by such motion. Suppose that at time $t$ the body is rotated back into original orientation. The equation (12) is also true when $\omega_{i j}$ is replaced by $\omega_{i j}+\Omega_{i j}$, where $\Omega_{i j}$ is a constant arbitrary skew-symmetric tensor. Thus, we find that the stress tensor $S_{i j}$ is symmetric. We denote by $E_{i j}$ the Lagrangian strain tensor

$$
E_{i j}=\frac{1}{2}\left(y_{k, i} y_{k, j}-\delta_{i j}\right)
$$

where $\delta_{i j}$ is the Kronecker delta. The equation of energy becomes

$$
\rho_{0} \dot{e}=S_{i j} \dot{E}_{i j}+\sigma_{i} \dot{\nu}_{1, i}+\tau_{i} \dot{\nu}_{2, i}-\xi \dot{\nu}_{1}-\zeta \dot{\nu}_{2}+Q_{j, j}+\rho_{0} S .
$$


The entropy production inequality is written in the form

$$
\int_{\Omega} \rho_{0} \dot{\eta} d v-\int_{\Omega} \frac{1}{T} \rho_{0} S d x-\int_{\partial \Omega} \frac{1}{T} Q d a \geq 0,
$$

for every part $\Omega$ of $B$ and every time. Here, $\eta$ is the entropy per unit mass, and $T$ is the temperature, which is assumed to be positive. If we introduce the Helmholtz free energy

$$
A=e-T \eta,
$$

then the equation (9) can be written as

$$
\rho_{0}(\dot{A}+\dot{T} \eta+T \dot{\eta})=S_{i j} \dot{E}_{i j}+\sigma_{i} \dot{\nu}_{1, i}+\tau_{i} \dot{\nu}_{2, i}-\xi \dot{\nu}_{1}-\zeta \dot{\nu}_{2}+Q_{j, j}+\rho_{0} S .
$$

A thermoelastic material with double porosity is defined as one for which the folowing constitutive equations hold

$$
\begin{aligned}
& A=\widetilde{A}(\mathcal{S}), S_{i j}=\widetilde{S}_{i j}(\mathcal{S}), \eta=\widetilde{\eta}(\mathcal{S}), Q_{i}=\widetilde{Q}_{i}(\mathcal{S}), \\
& \sigma_{i}=\widetilde{\sigma}_{i}(\mathcal{S}), \tau_{i}=\widetilde{\tau}_{i}(\mathcal{S}), \xi=\widetilde{\xi}(\mathcal{S}), \zeta=\widetilde{\zeta}(\mathcal{S}), \\
& \sigma=\widetilde{\sigma}\left(\mathcal{S}, n_{p}\right), \tau=\widetilde{\tau}\left(\mathcal{S}, n_{p}\right), Q=\widetilde{Q}\left(\mathcal{S}, n_{p}\right),
\end{aligned}
$$

where $\mathcal{S}=\left(E_{r s}, T, T_{, k}, \nu_{1}, \nu_{1, j}, \nu_{2}, \nu_{2, j}, x_{i}\right)$. The response functions are assumed to be sufficiently smooth. For a given deformation $\dot{\nu}_{1}$ and $\dot{\nu}_{2}$ in (8) may be chosen arbitrarily so that, on the basis of the constitutive equations we find

$$
\sigma=\sigma_{j} n_{j}, \quad \tau=\tau_{j} n_{j}, \quad Q=Q_{j} n_{j} .
$$

If we use (19) in (15) then we obtain the local form of the second law of thermodynamics

$$
\rho_{0} T \dot{\eta}-\rho_{0} S-Q_{j, j}+\frac{1}{T} Q_{j} T_{, j} \geq 0 .
$$

From (17), (18) and (20) we obtain

$$
\begin{aligned}
& \left(S_{i j}-\frac{\partial U}{\partial E_{i j}}\right) \dot{E}_{i j}-\left(\rho_{0} \eta+\frac{\partial U}{\partial T}\right) \dot{T}-\frac{\partial U}{\partial T_{, j}} T_{, j}+\left(\sigma_{j}-\frac{\partial U}{\partial \nu_{1, j}}\right) \dot{\nu}_{1, j}+ \\
& +\left(\tau_{j}-\frac{\partial U}{\partial \nu_{2, j}}\right) \dot{\nu}_{2, j}-\left(\xi+\frac{\partial U}{\partial \nu_{1}}\right) \dot{\nu}_{1}-\left(\zeta+\frac{\partial U}{\partial \nu_{2}}\right) \dot{\nu}_{2}+\frac{1}{T} Q_{j} T_{, j} \geq 0
\end{aligned}
$$

where $U=\rho_{0} A$. In absence of internal constraints, from (21) we get

$$
\begin{aligned}
& S_{i j}=\frac{\partial U}{\partial E_{i j}}, \rho_{0} \eta=-\frac{\partial U}{\partial T}, \sigma_{j}=\frac{\partial U}{\partial \nu_{1, j}}, \tau_{j}=\frac{\partial U}{\partial \nu_{2, j}}, \\
& \xi=-\frac{\partial U}{\partial \nu_{1}}, \zeta=-\frac{\partial U}{\partial \nu_{2}}, \frac{\partial U}{\partial T_{, k}}=0,
\end{aligned}
$$


and

$$
Q_{j} T_{, j} \geq 0 .
$$

We conclude that the constitutive equations become

$$
\begin{aligned}
& U=\widehat{U}\left(E_{r s}, T, \nu_{1}, \nu_{2}, \nu_{1, k}, \nu_{2, k}, x_{j}\right), \\
& S_{i j}=\frac{\partial U}{\partial E_{i j}}, \rho_{0} \eta=-\frac{\partial U}{\partial T}, \sigma_{j}=\frac{\partial U}{\partial \nu_{1, j}}, \tau_{j}=\frac{\partial U}{\partial \nu_{2, j}}, \\
& \xi=-\frac{\partial U}{\partial \nu_{1}}, \zeta=-\frac{\partial U}{\partial \nu_{2}}, Q_{i}=Q_{i}\left(E_{r s}, T, T_{, j}, \nu_{1}, \nu_{2}, \nu_{1, k}, \nu_{2, k}, x_{j}\right) .
\end{aligned}
$$

In view of (24) the energy equation (17) takes the form

$$
\rho_{0} T \dot{\eta}=Q_{j, j}+\rho_{0} S
$$

As in classical thermoelasticity, the inequality (23) implies that

$$
\widehat{Q}_{k}\left(E_{r s}, T, 0, \nu_{1}, \nu_{2}, \nu_{1, j}, \nu_{2, j}, x_{i}\right)=0 .
$$

The basic equations of the theory consist of the equations of motion (6) and (10), the equation of energy (25), the constitutive equations (24), and the geometrical equations (13). To the field equations we must adjoin boundary conditions and initial conditions. The initial conditions are

$$
\begin{aligned}
& y_{i}\left(x_{j}, 0\right)=y_{i}^{0}\left(x_{j}\right), \dot{y}_{i}\left(x_{j}, 0\right)=v_{i}^{0}\left(x_{j}\right), \eta\left(x_{j}, 0\right)=\eta^{0}\left(x_{j}\right), \\
& \nu_{\alpha}\left(x_{j}, 0\right)=\nu_{\alpha}^{0}\left(x_{j}\right), \dot{\nu}_{\alpha}\left(x_{j}, 0\right)=\zeta_{\alpha}^{0}\left(x_{j}\right),(\alpha=1,2),\left(x_{j}\right) \in \bar{B}
\end{aligned}
$$

where $y_{i}^{0}, v_{i}^{0}, \eta^{0}, \nu_{\alpha}^{0}$ and $\zeta_{\alpha}^{0}$ are prescribed functions. In the case of the first boundary value problem the boundary conditions are

$$
y_{i}=\widetilde{y}_{i}, T=\widetilde{T}, \nu_{\alpha}=\widetilde{\nu}_{\alpha},(\alpha=1,2), \text { on } \partial B \times\left(t_{0}, t_{1}\right),
$$

where $\widetilde{y}_{i}, \widetilde{T}$ and $\widetilde{\nu}_{\alpha}$ given. In the second boundary-value problem the boundary conditions are

$$
T_{j i} n_{j}=\widetilde{T}_{i}, Q_{j} n_{j}=\widetilde{Q}, \sigma_{j} n_{j}=\widetilde{\sigma}, \tau_{j} n_{j}=\widetilde{\tau} \text { on } \partial B \times\left(t_{0}, t_{1}\right),
$$

where the functions $\widetilde{T}_{i}, \widetilde{Q}, \widetilde{\sigma}$ and $\widetilde{\tau}$ are prescribed.

\section{LINEAR THEORY}

Let us introduce the notations

$$
u_{i}=y_{i}-x_{i}, \varphi=\nu_{1}-\nu_{1}^{*}, \psi=\nu_{2}-\nu_{2}^{*}, \theta=T-T_{0},
$$

where $T_{0}$ is the constant absolute temperature of the body in the reference configuration, and $\nu_{1}^{*}$ and $\nu_{2}^{*}$ are the volume fractions fields in the reference configuration. We assume 
that $u_{i}=\varepsilon u_{i}^{*}, \varphi=\varepsilon \varphi^{*}, \psi=\varepsilon \psi^{*}, \theta=\varepsilon \theta^{*}$ where $\varepsilon$ is a constant small enough for squares and higher powers to be neglected, and $u_{i}^{*}, \varphi^{*}, \psi^{*}$ and $\theta^{*}$ are independent of $\varepsilon$. In the linear theory the strain tensor $E_{i j}$ reduces to

$$
e_{i j}=\frac{1}{2}\left(u_{i, j}+u_{j, i}\right)
$$

The independent constitutive variables in the linear theory are $e_{i j}, \theta, \theta, k, \varphi, \psi, \varphi_{, j}$ and $\psi_{, j}$. We assume that the undeformed body is free from stresses and has zero intrinsic equilibrated body forces and entropy. In the case of centrosymmetric solids we have

$$
\begin{aligned}
2 U & =C_{i j r s} e_{i j} e_{r s}+2 B_{i j} e_{i j} \varphi+2 D_{i j} e_{i j} \psi-2 \beta_{i j} \theta e_{i j}+ \\
& +\alpha_{i j} \varphi_{, i} \varphi_{, j}+2 b_{i j} \varphi_{, i} \psi_{, j}+\gamma_{i j} \psi_{, i} \psi_{, j}+\alpha_{1} \varphi^{2}+ \\
& +\alpha_{2} \psi^{2}+2 \alpha_{3} \varphi \psi-2 \gamma_{1} \varphi \theta-2 \gamma_{2} \psi \theta-a \theta^{2}
\end{aligned}
$$

where the constitutive coefficients are prescribed functions of material coordinates. We have the symmetries

$$
C_{i j r s}=C_{r s i j}=C_{j i r s}, B_{i j}=B_{j i}, D_{i j}=D_{j i}, \beta_{i j}=\beta_{j i}, \alpha_{i j}=\alpha_{j i}, \gamma_{i j}=\gamma_{j i} .
$$

It follows from (11), (22) and (32) that in the linear theory the stress tensors $T_{i j}$ and $S_{i j}$ coincide. In this case we denote the stress tensor by $t_{i j}$. In view of $(22),(26)$, (30), (31) and (32) we obtain the following constitutive equations of the linear theory of centrosymmetric materials

$$
\begin{aligned}
t_{i j} & =C_{i j r s} e_{r s}+B_{i j} \varphi+D_{i j} \psi-\beta_{i j} \theta, \\
\sigma_{i} & =\alpha_{i j} \varphi_{, j}+b_{i j} \psi_{, j}, \\
\tau_{i} & =b_{j i} \varphi_{, j}+\gamma_{i j} \psi_{, j}, \\
\xi & =-B_{i j} e_{i j}-\alpha_{1} \varphi-\alpha_{3} \psi+\gamma_{1} \theta, \\
\zeta & =-D_{i j} e_{i j}-\alpha_{3} \varphi-\alpha_{2} \psi+\gamma_{2} \theta, \\
\rho_{0} \eta & =\beta_{i j} e_{i j}+\gamma_{1} \varphi+\gamma_{2} \psi+a \theta, \\
Q_{i} & =k_{i j} \theta_{, j} .
\end{aligned}
$$

From (23) and (34) we get

$$
k_{i j} \theta_{, i} \theta_{, j} \geq 0 .
$$

In the case of isotropic solids the constitutive equations have the form

$$
\begin{aligned}
t_{i j} & =\lambda e_{r r} \delta_{i j}+2 \mu e_{i j}+b \delta_{i j} \varphi+d \delta_{i j} \psi-\beta \delta_{i j} \theta, \\
\sigma_{i} & =\alpha \varphi_{, i}+b_{1} \psi_{, i}, \tau_{i}=b_{1} \varphi, i+\gamma \psi_{, i}, \\
\xi & =-b e_{j j}-\alpha_{1} \varphi-\alpha_{3} \psi+\gamma_{1} \theta, \zeta=-d e_{j j}-\alpha_{3} \varphi-\alpha_{2} \psi+\gamma_{2} \theta, \\
\rho_{0} \eta & =\beta e_{j j}+\gamma_{1} \varphi+\gamma_{2} \psi+a \theta, Q_{i}=k \theta, i
\end{aligned}
$$


where $\delta_{i j}$ is Kronecker's delta, and $\lambda, \mu, b, d, b_{1}, \alpha, \beta, \gamma, \alpha_{j}, \gamma_{\alpha}$ and $k$ are constitutive coefficients. In the context of the linear theory the equation of energy (25) takes the form

$$
\rho_{0} T_{0} \dot{\eta}=Q_{j, j}+\rho_{0} S
$$

The equations of motion (6) and (10) become

$$
\begin{aligned}
& t_{j i, j}+\rho_{0} f_{i}=\rho_{0} \ddot{u}_{i}, \\
& \sigma_{j, j}+\xi+\rho_{0} g=\kappa_{1} \ddot{\varphi}, \\
& \tau_{j, j}+\zeta+\rho_{0} \ell=\kappa_{2} \ddot{\psi} .
\end{aligned}
$$

The basic equations of the linear theory consist of the equations of motion (38), equation of energy (37), constitutive equations (34), and the geometrical equations (31). The first boundary-value problem is characterized by the boundary conditions

$$
u_{i}=\widetilde{u}_{i}, \varphi=\widetilde{\varphi}, \psi=\widetilde{\psi}, \theta=\widetilde{\theta} \text { on } \partial B \times\left(t_{0}, t_{1}\right),
$$

where $\widetilde{u}_{i}, \widetilde{\varphi}, \widetilde{\psi}$ and $\widetilde{\theta}$ are prescribed functions. In the case of the second boundary-value problem the boundary conditions are

$$
t_{j i} n_{j}=\widetilde{t}_{i}, \sigma_{i} n_{i}=\widetilde{\sigma}, \tau_{i} n_{i}=\widetilde{\tau}, Q_{j} n_{j}=\widetilde{Q} \text { on } \partial B \times\left(t_{0}, t_{1}\right),
$$

where the functions $\widetilde{t_{i}}, \widetilde{\sigma}, \widetilde{\tau}$ and $\widetilde{Q}$ are given. The initial conditions can be written in the form

$$
\begin{aligned}
& u_{i}\left(x_{j}, 0\right)=u_{i}^{0}\left(x_{j}\right), \dot{u}_{i}\left(x_{j}, 0\right)=v_{i}^{0}\left(x_{j}\right), \theta\left(x_{j}, 0\right)=\theta^{0}\left(x_{j}\right), \\
& \varphi\left(x_{j}, 0\right)=\varphi_{1}^{0}\left(x_{j}\right), \psi\left(x_{j}, 0\right)=\psi_{1}^{0}\left(x_{j}\right), \dot{\varphi}\left(x_{j}, 0\right)=\varphi_{2}^{0}\left(x_{j}\right), \\
& \dot{\psi}\left(x_{j}, 0\right)=\psi_{2}^{0}\left(x_{j}\right),\left(x_{j}\right) \in \bar{B},
\end{aligned}
$$

where $u_{i}^{0}, v_{i}^{0}, \theta^{0}, \varphi_{\alpha}^{0}$ and $\psi_{\alpha}^{0}$ are prescribed functions.

It follows from (31), (36), (38) and (37) that in the case of homogeneous and isotropic solids the functions $u_{j}, \varphi, \psi$ and $\theta$ satisfy the following equations

$$
\begin{aligned}
& \mu \Delta u_{i}+(\lambda+\mu) u_{j, j i}+b \varphi_{, i}+d \psi_{, i}-\beta \theta_{, i}+\rho_{0} f_{i}=\rho_{0} \ddot{u}_{i}, \\
& \alpha \Delta \varphi+b_{1} \Delta \psi-b u_{r, r}-\alpha_{1} \varphi-\alpha_{3} \psi+\gamma_{1} \theta+\rho_{0} g=\kappa_{1} \ddot{\varphi}, \\
& b_{1} \Delta \varphi+\gamma \Delta \psi-d u_{r, r}-\alpha_{3} \varphi-\alpha_{2} \psi+\gamma_{2} \theta+\rho_{0} \ell=\kappa_{2} \ddot{\psi}, \\
& k \Delta \theta-\beta T_{0} \dot{u}_{j, j}-\gamma_{1} T_{0} \dot{\varphi}-\gamma_{2} T_{0} \dot{\psi}-c \dot{\theta}=-\rho_{0} S,
\end{aligned}
$$

where $c=a T_{0}$. We note that in contrast with the classical theory of elastic materials with double structure, the porosity of the body in the equilibrium state depends on the displacement vector field. 


\section{INSTABILITY AND UNIQUENESS}

In this section we suppose that the body forces and heat supply are absent, and we consider the following boundary conditions

$$
u_{i}=0, \varphi=0, \psi=0, \theta=0 \text { on } \partial B \times\left(0, t_{1}\right) .
$$

The aim of this section is to propose a logarithmic convexity type argument to the equations (34), (37) and (38) with the initial conditions (41), and boundary conditions (43).

In the remains of the paper we assume that

(i) the mass density $\rho_{0}$, the thermal capacity $a$ and the functions $\kappa_{i}, i=1,2$ are strictly positive. That is:

$$
\rho_{0}\left(x_{j}\right) \geq \underline{\rho}>0 ; \quad a\left(x_{j}\right) \geq \underline{a}>0, \quad \kappa_{i}\left(x_{j}\right) \geq \underline{\kappa_{i}}>0, \quad i=1,2 .
$$

(ii) The thermal conductivity tensor $k_{i j}$ is positive definite. that is, there exists $k_{0}>0$ such that

$$
k_{i j} \xi_{i} \xi_{j} \geq k_{0} \xi_{i} \xi_{i} \text {, for every vector }\left(\xi_{i}\right) .
$$

(iii) The constitutive coefficients satisfy the symmetry relations (33);

We note that the physical meaning of condition (i) is clear. The condition (ii) is compatible with restriction (35) which shows that the heat conductivity tensor is nonnegative.

Logarithmic convexity argument is strongly based in the choice of a good function which satisfies several requirements. To define this function, it is useful to consider several preliminary relations. First, we integrate with respect to the time the energy equation. We have

$$
\int_{0}^{t} \frac{1}{T_{0}}\left(k_{i j} \theta_{, i}\right)_{, j} d s-\beta_{i j} e_{i j}-\gamma_{1} \varphi-\gamma_{2} \psi-a \theta=-\beta_{i j} u_{i, j}^{0}-\gamma_{1} \varphi_{1}^{0}-\gamma_{2} \psi_{1}^{0}-a \theta^{0} .
$$

We denote by $P\left(x_{j}\right)$ the function which is solution to the boundary value problem determined by the equation

$$
\frac{1}{T_{0}}\left(k_{i j} P_{, i}\right)_{, j}=\beta_{i j} u_{i, j}^{0}+\gamma_{1} \varphi_{1}^{0}+\gamma_{2} \psi_{1}^{0}+a \theta^{0},
$$

and the homogeneous boundary condition

$$
P=0 \text { on } \partial B \text {. }
$$

If we define the function $z=T+P$, where

$$
T=\int_{0}^{t} \theta d s
$$


we see that it satisfies the equation

$$
\frac{1}{T_{0}}\left(k_{i j} z_{, i}\right)_{, j}-\beta_{i j} e_{i j}-\gamma_{1} \varphi-\gamma_{2} \psi-a \theta=0 .
$$

To be used later we recall the energy equality

$$
\begin{gathered}
E(t)=\int_{B}\left(\rho_{0} \dot{u}_{i} \dot{u}_{i}+\kappa_{1}|\dot{\varphi}|^{2}+\kappa_{2}|\dot{\psi}|^{2}+a \theta^{2}+C_{i j k h} e_{i j} e_{k h}+\alpha_{i j} \varphi_{, i} \varphi_{, j}\right) d v \\
+\int_{B}\left(\gamma_{i j} \psi_{, i} \psi_{, j}+2 b_{i j} \varphi_{, i} \psi_{, j}+2 B_{i j} e_{i j} \varphi+2 D_{i j} e_{i j} \psi+\alpha_{1} \varphi^{2}+\alpha_{2} \psi^{2}+2 \alpha_{3} \varphi \psi\right) d v \\
+2 \int_{0}^{t} \int_{B} \frac{1}{T_{0}} k_{i j} \theta_{, i} \theta_{, j} d v d s=E(0) .
\end{gathered}
$$

This relation is satisfied for every solution of our problem. We introduce the function

$$
F_{h, \omega}(t)=\int_{B}\left(\rho_{0} u_{i} u_{i}+\kappa_{1} \varphi^{2}+\kappa_{2} \psi^{2}\right) d v+\int_{0}^{t} \int_{B} \frac{1}{T_{0}} k_{i j} z_{, i} z_{, j} d v d s+h(t+\omega)^{2} .
$$

Here $h$ and $\omega$ are two positive constants to be determined later. We see that

$$
\begin{gathered}
\dot{F}_{h, \omega}(t)=2 \int_{B}\left(\rho_{0} u_{i} \dot{u}_{i}+\kappa_{1} \varphi \dot{\varphi}+\kappa_{2} \psi \dot{\psi}\right) d v+\int_{0}^{t} \int_{B} \frac{2}{T_{0}} k_{i j} z_{, i} \dot{z}_{, j} d v d s \\
+2 h(t+\omega)-\int_{B} \frac{1}{T_{0}} k_{i j} P_{, i} P_{, j} d v
\end{gathered}
$$

and

$\ddot{F}_{h, \omega}(t)=2 \int_{B}\left(\rho_{0}\left(u_{i} \ddot{u}_{i}+\dot{u}_{i} \dot{u}_{i}\right)+\kappa_{1}(\varphi \ddot{\varphi}+\dot{\varphi} \dot{\varphi})+\kappa_{2}(\psi \ddot{\psi}+\dot{\psi} \dot{\psi})\right) d v+\int_{B} \frac{2}{T_{0}} k_{i j} z_{, i} \dot{z}_{, j} d v+2 h$.

In view of the evolutionary equations and the divergence theorem, we can write

$$
\begin{gathered}
\ddot{F}_{h, \omega}(t)=2 \int_{B}\left(\rho_{0} \dot{u}_{i} \dot{u}_{i}+\kappa_{1} \dot{\varphi} \dot{\varphi}+\kappa_{2} \dot{\psi} \dot{\psi}-C_{i j k l} e_{i j} e_{k h}-\alpha_{i j} \varphi_{, i} \varphi_{, j}-\gamma_{i j} \psi_{, i} \psi_{, j}-2 b_{i j} \varphi_{, i} \psi_{, j}\right) d v \\
-2 \int_{B}\left(B_{i j} e_{i j} \varphi+D_{i j} e_{i j} \psi+\alpha_{1} \phi^{2}+\alpha_{2} \psi^{2}+2 \alpha_{3} \varphi \psi\right) d v \\
-\int_{B} \frac{2}{T_{0}}\left(\left(k_{i j} z_{, i}\right)_{, j}-\beta_{i j} e_{i j}-\gamma_{1} \varphi-\gamma_{2} \psi\right) \dot{z} d v+2 h
\end{gathered}
$$

Using again the field equations, we see that

$$
\ddot{F}_{h, \omega}(t)=2 \int_{B}\left(\rho_{0} \dot{u}_{i} \dot{u}_{i}+\kappa_{1} \dot{\varphi} \dot{\varphi}+\kappa_{2} \dot{\psi} \dot{\psi}-C_{i j k l} e_{i j} e_{k h}-\alpha_{i j} \varphi_{, i} \varphi_{, j}-\gamma_{i j} \psi_{, i} \psi_{, j}-2 b_{i j} \varphi_{, i} \psi_{, j}\right) d v
$$




$$
-2 \int_{B}\left(B_{i j} e_{i j} \varphi+D_{i j} e_{i j} \psi+\alpha_{1} \phi^{2}+\alpha_{2} \psi^{2}+2 \alpha_{3} \varphi \psi\right) d v-2 \int_{B} a|\dot{z}|^{2} d v+2 h .
$$

Using the energy equality we find that

$$
\ddot{F}_{h, \omega}(t)=4 \int_{B}\left(\rho_{0} \dot{u}_{i} \dot{u}_{i}+\kappa_{1} \dot{\varphi} \dot{\varphi}+\kappa_{2} \dot{\psi} \dot{\psi}\right) d v+4 \int_{0}^{t} \int_{B} \frac{1}{T_{0}} k_{i j} \theta_{, i} \theta_{, j} d v-2(E(0)-h) .
$$

If we denote by

$$
\nu=\frac{2}{T_{0}} \int_{B} k_{i j} P_{, i} P_{, j} d v
$$

we obtain that the inequality

$$
F_{h, \omega} \ddot{F}_{h, \omega}-\left(\dot{F}_{h, \omega}-\nu\right)^{2} \geq 2(h+E(0)) F_{h, \omega},
$$

holds.

This inequality is well known. In case of null initial data, we have that $\nu=0$. Our inequality with $h=\omega=0$ becomes

$$
F \ddot{F}-(\dot{F})^{2} \geq 0
$$

where we denote by $F(t)$ the function $F_{0,0}(t)$. From this inequality we derive that

$$
F(t) \leq F(0)^{1-t / t_{1}} F\left(t_{1}\right)^{t / t_{1}}, \quad 0 \leq t \leq t_{1},
$$

and we conclude that $F(t)=0,0 \leq t \leq t_{1}$. From this relation we get a uniqueness result.

In the general case and assuming that $E(0)<0$, we can always take $\omega$ so large to guarantee that $\dot{F}_{h, \omega}(0)>\nu$, and then we obtain that

$$
F_{h, \omega}(t) \geq \frac{F_{h, \omega}(0) \dot{F}_{h, \omega}(0)}{\dot{F}_{h, \omega}(0)-\nu} \exp \left(\frac{\dot{F}_{h, \omega}(0)-\nu}{F_{h, \omega}(0)} t\right)-\frac{\nu F_{h, \omega}(0)}{\dot{F}_{h, \omega}(0)-\nu}
$$

This inequality gives the exponential growth of the solutions. We have proved that:

Theorem 1. Assume that conditions (i)-(iii) hold. Then

(I) The first initial-boundary-value problem has at most one solution.

(II) If $E(0)<0$, then the solution becomes unbounded in an exponential way.

It is worth noting that a suitable variation in the logarithmic convexity argument allows us to obtain Holder stability of the solutions in a similar way to the one proposed by Straughan [13] for the classical theory. 


\section{AN EXISTENCE THEOREM}

In this section we consider a semigroup approach to obtain an existence result in the dynamical theory, with suitable initial and boundary conditions. From now on, we assume the boundary conditions (43)

We introduce the quadratic form $W$ defined by

$$
\begin{aligned}
2 W & =C_{i j r s} e_{i j} e_{r s}+2 B_{i j} e_{i j} \varphi+2 D_{i j} e_{i j} \psi+ \\
& +\alpha_{i j} \varphi_{, i} \varphi_{, j}+2 b_{i j} \varphi_{, i} \psi_{, j}+\gamma_{i j} \psi_{, i} \psi_{, j}+\alpha_{1} \varphi^{2}+\alpha_{2} \psi^{2}+2 \alpha_{3} \varphi \psi .
\end{aligned}
$$

In addition to the assumptions (i)-(iii) we assume that:

(iv) $W$ is a positive definite quadratic form. That is, there exists a positive constant $C_{0}$ such that

$$
W \geq C_{0}\left(e_{i j} e_{i j}+|\nabla \varphi|^{2}+|\nabla \psi|^{2}+\varphi^{2}+\psi^{2}\right) .
$$

In the case of homogeneous and isotropic materials this condition holds if and only if the constitutive constants satisfy the following inequalities (see the appendix)

$$
\begin{gathered}
\mu>0,3 \lambda+2 \mu>0, \quad \alpha_{2}>0, \quad \alpha_{1} \alpha_{2}-\alpha_{3}^{2}>0, \\
(3 \lambda+2 \mu)\left(\alpha_{1} \alpha_{2}-\alpha_{3}^{2}\right)>3\left(\alpha_{1} d^{2}+\alpha_{2} b^{2}-2 \alpha_{3} b d\right), \quad \alpha>0, \quad \alpha \gamma>b_{1}^{2} .
\end{gathered}
$$

The condition (60) is related with the stability of the dynamical problem. In fact, we have seen in the previous section that in case that this condition does not hold the dynamical problem becomes unstable.

Let $W_{0}^{2,2}$ and $L^{2}$ be the usual Hilbert spaces and denote

$$
\mathcal{Z}=\left\{(\mathbf{u}, \mathbf{v}, \varphi, \zeta, \psi, \chi, \theta), \mathbf{u} \in \mathbf{W}_{0}^{2,2}(B), \mathbf{v} \in \mathbf{L}^{2}(B), \varphi, \psi \in W_{0}^{2,2}(B), \zeta, \chi, \theta \in L^{2}(B)\right\},
$$

where $\mathbf{W}_{0}^{2,2}=\left[W_{0}^{2,2}\right]^{3}$ and $\mathbf{L}^{2}=\left[L^{2}\right]^{3}$. We introduce the operators

$$
\begin{aligned}
M_{i} \mathbf{u} & =\rho_{0}^{-1}\left(C_{j i r s} e_{r s}\right)_{, j}, N_{i} \varphi=\rho_{0}^{-1}\left(B_{i j} \varphi\right)_{, j}, N_{i}^{*} \psi=\rho_{0}^{-1}\left(D_{i j} \psi\right)_{, j}, P_{i} \theta=\rho_{0}^{-1}\left(-\beta_{j i} \theta_{, i}\right)_{, j}, \\
R \mathbf{u} & =-\kappa_{1}^{-1} B_{i j} e_{i j}, S \varphi=\kappa_{1}^{-1}\left[\left(\alpha_{i j} \varphi, i\right)_{, j}-\alpha_{1} \varphi\right], T \psi=\kappa_{1}^{-1}\left[\left(\beta_{i j} \psi, i\right)_{, j}-\alpha_{3} \psi\right], \\
U \theta & =\kappa_{1}^{-1} \gamma_{1} \theta, V \mathbf{u}=-\kappa_{2}^{-1} D_{i j} e_{i j}, W \varphi=\kappa_{2}^{-1}\left[\left(b_{i j} \varphi, i\right)_{, j}-\alpha_{3} \varphi\right], X \psi=\left(\gamma_{i j} \psi_{, i}\right)_{, j}-\alpha_{2} \psi, \\
Y \theta & =\kappa_{2}^{-1} \gamma_{2} \theta, Q \mathbf{v}=a^{-1}\left(-\beta_{i j} f_{i j}\right), L \zeta=-a^{-1} \gamma_{1} \zeta, G \chi=-a^{-1} \gamma_{2} \chi, R^{*} \theta=\left(a T_{0}\right)^{-1}\left(k_{i j} \theta_{, i}\right)_{, j} .
\end{aligned}
$$

Let us consider the matrix operator $\mathcal{A}$ defined on $\mathcal{Z}$ by

$$
\left(\begin{array}{ccccccc}
\mathbf{0} & \mathbf{I d} & \mathbf{0} & \mathbf{0} & \mathbf{0} & \mathbf{0} & \mathbf{0} \\
\mathbf{M} & \mathbf{0} & \mathbf{N} & \mathbf{0} & \mathbf{N}^{*} & \mathbf{0} & \mathbf{P} \\
0 & 0 & 0 & I d & 0 & 0 & 0 \\
R & 0 & S & 0 & T & 0 & U \\
0 & 0 & 0 & 0 & 0 & I d & 0 \\
V & 0 & W & 0 & X & 0 & Y \\
0 & Q & 0 & L & 0 & G & R^{*}
\end{array}\right),
$$


where $\mathbf{M}=\left(M_{i}\right), \mathbf{N}=\left(N_{i}\right), \mathbf{N}^{*}=\left(N_{i}^{*}\right)$ and $\mathbf{P}=\left(P_{i}\right)$. The domain $\mathcal{D}$ of the operator $\mathcal{A}$ contains the set

$$
\mathbf{W}_{0}^{1,2} \cap \mathbf{W}^{2,2} \times \mathbf{W}_{0}^{1,2} \times W_{0}^{1,2} \cap W^{2,2} \times W_{0}^{1,2} \times W_{0}^{1,2} \cap W^{2,2} \times W_{0}^{1,2} \times W^{2,2},
$$

which is a dense subspace of the Hilbert space $\mathcal{Z}$.

The boundary-initial-value problem can be transformed into the following abstract equation in the space $\mathcal{Z}$

$$
\frac{d \omega}{d t}=\mathcal{A} \omega+G(t), \quad \omega(0)=\omega_{0},
$$

where $G(t)=\left(\mathbf{0}, \mathbf{f}, 0, \kappa_{1}^{-1} \rho_{0} g, 0, \kappa_{2}^{-1} \rho_{0} l, a^{-1} \rho_{0} S\right), \omega_{0}=\left(\mathbf{u}^{0}, \mathbf{v}^{0}, \varphi_{1}^{0}, \varphi_{2}^{0}, \psi_{1}^{0}, \psi_{2}^{0}, \theta^{0}\right)$. Let $\omega=(\mathbf{u}, \mathbf{v}, \varphi, \zeta, \psi, \chi, \theta)$ and $\omega^{\prime}=\left(\mathbf{u}^{\prime}, \mathbf{v}^{\prime}, \varphi^{\prime}, \zeta^{\prime}, \psi^{\prime}, \chi^{\prime}, \theta^{\prime}\right)$. We introduce the inner product

$$
<\omega, \omega^{\prime}>=\int_{B}\left(\rho v_{i} v_{i}^{\prime}+\kappa_{1} \zeta \zeta^{\prime}+\kappa_{2} \chi \chi^{\prime}+a \theta \theta^{\prime}+2 W^{*}\right) d v
$$

where

$$
\begin{gathered}
2 W^{*}=C_{i j r s} e_{i j} e_{r s}^{\prime}+\alpha_{i j} \varphi_{, i} \varphi_{, j}^{\prime}+\gamma_{i j} \psi_{, i} \psi_{, j}^{\prime}+b_{i j}\left(\varphi_{, i} \psi_{, j}^{\prime}+\varphi_{, i}^{\prime} \psi_{, j}\right)+B_{i j}\left(e_{i j} \varphi^{\prime}+e_{i j}^{\prime} \varphi\right) \\
+D_{i j}\left(e_{i j} \psi^{\prime}+e_{i j}^{\prime} \psi\right)+\alpha_{1} \varphi \varphi^{\prime}+\alpha_{2} \psi \psi^{\prime}+\alpha_{3}\left(\varphi \psi^{\prime}+\varphi^{\prime} \psi\right) .
\end{gathered}
$$

It is worth noting that this inner product defines the norm

$$
\|\omega\|^{2}=\int_{B}\left(\rho v_{i} v_{i}+\kappa_{1} \zeta^{2}+\kappa_{2} \chi^{2}+a \theta^{2}+2 W\right) d v,
$$

where $W$ has been defined at (59).

This norm is equivalent to the usual norm in $\mathcal{Z}$. We also note that for every $\omega \in \mathcal{D}$, we have

$$
<\mathcal{A} \omega, \omega>=-\frac{1}{2 T_{0}} \int_{B} k_{i j} \theta_{, i} \theta_{, j} d v \leq 0 .
$$

Lemma 1. Suppose that conditions (i)-(iv) hold. Let $\rho(\mathcal{A})$ be the resolvent of $\mathcal{A}$. Then, $0 \in \rho(\mathcal{A})$.

Proof. Let us show that we can find $\omega=(\mathbf{u}, \mathbf{v}, \varphi, \zeta, \psi, \chi, \theta) \in \mathcal{D}$ such that

$$
\mathcal{A} \omega=\mathcal{F},
$$

for any $\mathcal{F}=\left(\mathbf{f}_{1}, \mathbf{f}_{2}, f_{3}, f_{4}, f_{5}, f_{6}, f_{7}\right) \in \mathcal{Z}$. In terms of the components we get

$$
\begin{gathered}
\mathbf{v}=\mathbf{f}_{1}, \mathbf{M u}+\mathbf{N} \varphi+\mathbf{N}^{*} \psi+\mathbf{P} \theta=\rho \mathbf{f}_{2}, \zeta=f_{3} R \mathbf{u}+S \varphi+T \psi+U \theta=\kappa_{1} f_{4} \\
\chi=f_{5} V \mathbf{u}+W \varphi+X \psi+Y \theta=\kappa_{2} f_{6} \quad Q \mathbf{v}+L \zeta+G \chi+R^{*} \theta=a f_{7} .
\end{gathered}
$$

From these equations we see that $\mathbf{v} \in \mathbf{W}_{0}^{1,2}$ and $\chi, \zeta \in W_{0}^{1,2}$ and we can write

$$
R^{*} \theta=a f_{7}-Q \mathbf{f}_{1}-L f_{3}-G f_{5} .
$$


As the right hand side of this equation belongs to $W^{-1,2}$, we see that there exists a solution $\theta \in W_{0}^{1,2}$. We then obtain the system

$\mathbf{M u}+\mathbf{N} \varphi+\mathbf{N}^{*} \psi=\rho \mathbf{f}_{2}-\mathbf{P} \theta, R \mathbf{u}+S \varphi+T \psi=\kappa_{1} f_{4}-U \theta, V \mathbf{u}+W \varphi+X \psi=\kappa_{2} f_{6}-Y \theta$.

To solve this system we define the bilinear form:

$$
\mathcal{B}\left[(\mathbf{u}, \varphi, \psi),\left(\mathbf{u}^{*}, \varphi^{*}, \psi^{*}\right)\right]=I,
$$

where

$$
I=\int_{B}\left(\left(\mathbf{M u}+\mathbf{N} \varphi+\mathbf{N}^{*} \psi\right) \mathbf{u}^{*}+(R \mathbf{u}+S \varphi+T \psi) \varphi^{*}+(V \mathbf{u}+W \varphi+X \psi) \psi^{*}\right) d v .
$$

After the use of the divergence theorem we see that this is a bounded bilinear form defined in $\mathbf{W}^{1,2}$. In view of the condition (60) it is coercive. On the other side the right-hand side belongs to $\mathbf{W}^{-1,2} \times W^{-1,2} \times W^{-1,2}$. The existence of solution for this system is guarantee because of the Lax-Milgran theorem. Consequently, there exists $\mathbf{u}, \varphi, \psi \in \mathbf{W}_{0}^{1,2}$ satisfying the system (69). Thus, we conclude that the equation (66) has a solution in the domain $\mathcal{D}$ and the theorem is proved.

Theorem 2. Suppose that hypotheses (i)-(iv) hold. Then the operator $\mathcal{A}$ is the generator of a $C^{0}$-semigroup of contractions in the Hilbert space $\mathcal{Z}$.

Proof. The proof is a direct consequence of the Lumer-Phillips theorem, since the operator $\mathcal{A}$ is dissipative, with a dense domain and $0 \in \rho(\mathcal{A})$ (see for example Liu and Zheng, [28]).

Now, we can state the main result of this section.

Theorem 3. Suppose that hypotheses (i)-(iv) hold. Let $G(t) \in C^{1}\left(\mathbb{R}^{+}, \mathcal{Z}\right) \cap C^{0}\left(\mathbb{R}^{+}, \mathcal{D}\right)$ and $\omega_{0} \in \mathcal{D}$. Then, there exists a unique solution $\omega(t) \in C^{1}\left(\mathbb{R}^{+}, \mathcal{Z}\right) \cap C^{0}\left(\mathbb{R}^{+}, \mathcal{D}\right)$ to the problem (62).

Remark. The existence of a $C^{0}$-semigroup implies the continuous dependence with respect initial data and supply terms. As we have seen the existence, uniqueness and continuous dependence of solutions, we have proved that the problem is well posed in the sense of Hadamard. Furthermore, as we assume that the internal energy is positive we see that

$$
\|\omega(t)\| \leq E(0),
$$

for every $t$ in the case of the homogeneous problem. This gives the stability of the solutions in the case that (i)-(iv) hold. In view of the results in the previous section, we see that the positive definiteness of the internal energy plays a fundamental role to guarantee the stability of the solutions of the homogeneous problem.

\section{ELASTIC SPACE WITH A SPHERICAL CAVITY}

In this section we study the equilibrium of an unbounded medium with a spherical cavity of radius $a$, with center at the origin of coordinates. We assume that the body 
forces and the heat source are absent and that the boundary of the cavity is subjected to a constant temperature $T^{*}$ and is free of traction. As we have pointed out before, in our theory the porosity structure is influenced by the displacement field in the static case. Therefore the analysis will be more difficult than the one corresponding to the classical theory.

In absence of time dependence, the equations (41) reduce to

$$
\begin{aligned}
& \mu \Delta u_{i}+(\lambda+\mu) u_{j, j i}+b \varphi_{, i}+d \psi_{, i}=\beta \theta_{, i}, \\
& \alpha \Delta \varphi+b_{1} \Delta \psi-b u_{j, j}-\alpha_{1} \varphi-\alpha_{3} \psi=-\gamma_{1} \theta, \\
& b_{1} \Delta \varphi+\gamma \Delta \psi-d u_{j, j}-\alpha_{3} \varphi-\alpha_{2} \psi=-\gamma_{2} \theta,
\end{aligned}
$$

and

$$
\Delta \theta=0 .
$$

We introduce the notation $r=\left(x_{1}^{2}+x_{2}^{2}+x_{3}^{2}\right)^{1 / 2}$. On the boundary of cavity we have the conditions

$$
t_{j i} n_{j}=0, \quad \sigma_{i} n_{i}=0, \quad \tau_{i} n_{i}=0, \quad \theta=T^{*} \text { on } r=a .
$$

The conditions at infinity are

$$
\begin{aligned}
& u_{i}=O(1), u_{i, j}=O\left(r^{-1}\right), \theta=O\left(r^{-1}\right), \theta_{, j}=o\left(r^{-1}\right), \varphi=O\left(r^{-1}\right), \varphi_{, j}=o\left(r^{-1}\right), \\
& \psi=O\left(r^{-1}\right), \psi_{, j}=o\left(r^{-1}\right) .
\end{aligned}
$$

The conditions at infinity for the displacement vector are the same as in classical thermoelasticity. The solution of the equation (71) which satisfies the condition on the boundary of cavity and the conditions at infinity is given by

$$
\theta=\frac{a}{r} T^{*}
$$

We seek the solution for the system of equations (70) in the form

$$
u_{i}=\frac{\partial G(r)}{\partial x_{i}}, \varphi=\Phi(r), \psi=\Psi(r),
$$

where $G, \Phi$ and $\Psi$ are unknown functions. The equations (70) are satisfied if the functions $G, \Phi$ and $\Psi$ satisfy the equations

$$
\begin{aligned}
& (\lambda+2 \mu) \Delta G+b \Phi+d \Psi=\beta \theta, \\
& \left(\alpha \Delta-\alpha_{1}\right) \Phi+\left(b_{1} \Delta-\alpha_{3}\right) \Psi-b \Delta G=-\gamma_{1} \theta, \\
& \left(b_{1} \Delta-\alpha_{3}\right) \Phi+\left(\gamma \Delta-\alpha_{2}\right) \Psi-d \Delta G=-\gamma_{2} \theta .
\end{aligned}
$$

From the first equation we get

$$
\Delta G=\kappa(\beta \theta-b \Phi-d \Psi)
$$


where $\kappa=1 /(\lambda+2 \mu)$. We introduce the notations

$$
L_{11}=\alpha \Delta-\alpha_{1}^{*}, L_{12}=b_{1} \Delta-\alpha_{2}^{*}, L_{22}=\gamma \Delta-\alpha_{3}^{*},
$$

where the constants $\alpha_{j}^{*}$ are defined by

$$
\alpha_{1}^{*}=\alpha_{1}-b^{2} \kappa, \alpha_{2}^{*}=\alpha_{2}-\kappa d^{2}, \alpha_{3}^{*}=\alpha_{3}-\kappa b d .
$$

In view of (76) and (77) we find that the functions $\Phi$ and $\Psi$ satisfy the equations

$$
L_{11} \Phi+L_{12} \Psi=-s_{1} \theta, \quad L_{12} \Phi+L_{22} \Psi=-s_{2} \theta .
$$

Here we have used the notations

$$
s_{1}=\gamma_{1}-\kappa \beta b, s_{2}=\gamma_{2}-\kappa \beta d .
$$

We seek the solution of the system (80) in the form

$$
\Phi=\Phi_{0}+\chi_{1} \theta, \quad \Psi=\Psi_{0}+\chi_{2} \theta,
$$

where $\chi_{1}=\left(s_{1} \alpha_{2}^{*}-s_{2} \alpha_{3}^{*}\right) / d_{1}, \chi_{2}=\left(s_{2} \alpha_{1}^{*}-s_{1} \alpha_{3}^{*}\right) / d_{1}, d_{1}=\alpha_{1}^{*} \alpha_{2}^{*}-\left(\alpha_{3}^{*}\right)^{2}$. It follows from (80) and (82) that the functions $\Phi_{0}$ and $\Psi_{0}$ satisfy the equations

$$
L_{11} \Phi_{0}+L_{12} \Psi_{0}=0, L_{12} \Phi_{0}+L_{22} \Psi_{0}=0 .
$$

We introduce the notation

$$
D=L_{11} L_{22}-L_{12}^{2}
$$

Let

$$
\Phi_{0}=L_{22} V, \quad \Psi_{0}=-L_{12} V,
$$

where the function $V$ satisfies the equation

$$
D V=0 .
$$

Then $\Phi_{0}$ and $\Psi_{0}$ satisfy the equations (83). We can prove this assertion by substituting the functions $\Phi_{0}$ and $\Psi_{0}$ from (85) into the system (83). The operator $D$ can be written in the form

$$
D=\left(\alpha \gamma-b_{1}^{2}\right)\left(\Delta-k_{1}^{2}\right)\left(\Delta-k_{2}^{2}\right),
$$

where $k_{1}^{2}$ and $k_{2}^{2}$ are the roots of the equation

$$
\left(\alpha \gamma-b_{1}^{2}\right) x^{2}-\left(\alpha_{1}^{*} \gamma+\alpha_{2}^{*} \alpha-2 b_{1} \alpha_{3}^{*}\right) x+\alpha_{1}^{*} \alpha_{2}^{*}-\left(\alpha_{3}^{*}\right)^{2}=0 .
$$

We note that the positive definiteness of the internal energy implies that $\alpha \gamma-b_{1}^{2}>0$.

The function $V$ satisfies the equation

$$
\left(\Delta-k_{1}^{2}\right)\left(\Delta-k_{2}^{2}\right) V=0 .
$$


We can write $V=V_{1}+V_{2}$ where the functions $V_{1}$ and $V_{2}$ satisfy the equations

$$
\left(\Delta-k_{1}^{2}\right) V_{1}=0,\left(\Delta-k_{2}^{2}\right) V_{2}=0 .
$$

Let us assume that $k_{1}$ and $k_{2}$ are distinct positive constants. The other cases can be studied in a similar way. The functions $V_{1}$ and $V_{2}$ that satisfy the equations (89) and the conditions at infinity are given by

$$
V_{1}=C_{1} r^{-1} e^{-k_{1} r}, \quad V_{2}=C_{2} r^{-1} e^{-k_{2} r},
$$

where $C_{1}$ and $C_{2}$ are arbitrary constants. Thus, we get

$$
V=r^{-1}\left(C_{1} e^{-k_{1} r}+C_{2} e^{-k_{2} r}\right) .
$$

It follows from (82), (85), (89) and (90) that the functions $\Phi$ and $\Psi$ have the form

$$
\begin{aligned}
& \Phi=r^{-1}\left[p_{1} C_{1} e^{-k_{1} r}+p_{2} C_{2} e^{-k_{2} r}+\chi_{1} a T^{*}\right], \\
& \Psi=r^{-1}\left[\pi_{1} C_{1} e^{-k_{1} r}+\pi_{2} C_{2} e^{-k_{2} r}+\chi_{2} a T^{*}\right],
\end{aligned}
$$

where $p_{\beta}=\gamma k_{\beta}^{2}-\alpha_{3}^{*}$ and $\pi_{\beta}=\alpha_{2}^{*}-b_{1} k_{\beta}^{2},(\beta=1,2)$. Let us denote

$$
m_{\alpha}=b p_{\alpha}+d \pi_{\alpha},(\alpha=1,2), 2 \beta^{*}=\beta-b \chi_{1}-d \chi_{2} .
$$

In view of (74), (89) and (91), from (77) we obtain

$$
G=C_{3} r^{-1}+\kappa\left(a \beta^{*} T^{*} r-m_{1} k_{1}^{-2} C_{1} r^{-1} e^{-k_{1} r}-m_{2} k_{2}^{-2} C_{2} r^{-1} e^{-k_{2} r}\right),
$$

where $C_{3}$ is an arbitrary constant. From (35) and (75) we find

$$
\begin{aligned}
& t_{i j}=\lambda \delta_{i j} \Delta G+2 \mu G_{, i j}+(b \Phi+d \Psi-\beta \theta) \delta_{i j}, \\
& \sigma_{i}=\left(\alpha \Phi^{\prime}+b_{1} \Psi^{\prime}\right) x_{i} r^{-1}, \tau_{i}=\left(b_{1} \Phi^{\prime}+\gamma \Psi^{\prime}\right) x_{i} r^{-1},
\end{aligned}
$$

where $f^{\prime}=d f / d r$. On the boundary $r=a$ we have

$$
\begin{aligned}
& t_{j i} n_{j}=x_{i} a^{-1}\left[\lambda \Delta G+2 \mu G^{\prime \prime}+b \Phi+d \psi-\beta \theta\right], \\
& \sigma_{i} n_{i}=\alpha \Phi^{\prime}+b_{1} \Psi^{\prime} \\
& \tau_{i} n_{i}=b_{1} \Phi^{\prime}+\gamma \Psi^{\prime} .
\end{aligned}
$$

If we use (91) and (93), then the relations (95) become

$$
\begin{aligned}
t_{j i} n_{j} & =x_{i} a^{-1}\left\{4 \mu C_{3} a^{-3}+\left[\zeta_{1} a^{-1}-2 \mu \Gamma_{1}(a)\right] C_{1} e^{-k_{1} a}+\right. \\
& \left.+\left[\zeta_{2} a^{-1}-2 \mu \Gamma_{2}(a)\right] C_{2} e^{-k_{2} a}+\zeta_{3} T^{*}\right\}, \\
\sigma_{i} n_{i} & =-a^{-1}\left\{\left(k_{1}+a^{-1}\right) S_{11} C_{1} e^{-k_{1} a}+\left(k_{2}+a^{-1}\right) S_{12} C_{2} e^{-k_{2} a}-\left(\chi_{1} \alpha+\chi_{2} b_{1}\right) T^{*}\right\}, \\
\tau_{i} n_{i} & =-a^{-1}\left\{\left(k_{1}+a^{-1}\right) S_{21} C_{1} e^{-k_{1} a}+\left(k_{2}+a^{-1}\right) S_{22} C_{2} e^{-k_{2} a}-\left(\chi_{1} \alpha+\chi_{2} \gamma\right) T^{*}\right\},
\end{aligned}
$$


where we have used the notations

$$
\begin{aligned}
& \Gamma_{\alpha}(r)=\kappa m_{\alpha}\left(2 k_{\alpha}^{-2} r^{-3}+2 k_{\alpha}^{-3} r^{-2}+k_{\alpha}^{-4} r^{-1}\right),(\text { no sum } ; \alpha=1,2), \\
& \zeta_{\alpha}=\left(p_{\alpha} b+\pi_{\alpha} d\right)(1-\lambda \kappa), \zeta_{3}=(1-\lambda \kappa)\left(\chi_{1} b+\chi_{2} d-\beta\right), \\
& S_{1 \rho}=\alpha p_{\rho}+b_{1} \pi_{\rho}, S_{2 \rho}=b_{1} p_{\rho}+\pi_{\rho} \gamma .
\end{aligned}
$$

In view of (96), the conditions $\sigma_{i} n_{i}=0$ and $\tau_{i} n_{i}=0$ on $r=a$, imply that the constants $C_{1}$ and $C_{2}$ are given by

$$
\begin{aligned}
& C_{1}=\frac{a T^{*} e^{k_{1} a}}{\vartheta\left(1+k_{1} a\right)}\left[S_{22}\left(\chi_{1} \alpha+\chi_{2} b_{1}\right)-S_{12}\left(\chi_{1} b_{1}+\chi_{2} \gamma\right)\right], \\
& C_{2}=\frac{a T^{*} e^{k_{1} a}}{\vartheta\left(1+k_{1} a\right)}\left[S_{11}\left(\chi_{1} b_{1}+\chi_{2} \gamma\right)-S_{21}\left(\chi_{1} \alpha+\chi_{2} b_{1}\right)\right], \\
& \vartheta=S_{11} S_{22}-S_{12} S_{21} .
\end{aligned}
$$

From the condition $t_{j i} n_{j}=0$ on the boundary of cavity we determine the constant $C_{3}$,

$$
C_{3}=\frac{a^{2}}{4 \pi}\left\{\left[2 a \mu \Gamma_{1}(a)-\zeta_{1}\right] C_{1} e^{-k_{1} a}+\left[2 a \mu \Gamma_{2}(a)-\zeta_{2}\right] C_{2} e^{-k_{2} a}-a \zeta_{3} T^{*}\right\} .
$$

The solution of the problem is given by (75), (91) and (93). The results established here can be used in order to obtain in the solution in the case of a thick walled spherical shell.

\section{References}

1. G. I. Barrenblatt, I. P. Zheltov and I. N. Kockina, Basic Concepts in the Theory of Seepage of Homogeneous Liquids in Fissured Rocks (Strata), Prikl.Mat. Mekh. (English Translation), vol. 24, pp.1286-1303, 1960.

2. G. I. Barenblatt and I. P. Zheltov, On the Basic Equations of Seepage of Homogeneous Liquids in Fissured Rock, Akad. Nauk SSSR (English Translation), vol. $132,545-548,1960$.

3. R. Wilson and E. Aifantis, On the Theory of Consolidation with Double Porosity, Int. J. Eng. Sci.,vol. 20 , pp. 1009-1035, 1982.

4. N. Khalili and S. Valliappan, Unified Theory of Flow and Deformation in Double Porous Media, Eur. J. Mech. A, Solids, vol. 15, pp. 321-336,1996.

5. I. Masters, I.,W.K.S. Pao and R.W. Lewis,Coupling Temperature to a Doubleporosity Model of Deformable Porous Media, Int. J. Numer. Methods Eng., vol. 49, pp. 421-438, 2000. 
6. J.G. Berryman and H.F. Wang, Elastic Wave Propagation and Attenuation in a Double - porosity Dual - permeability Medium, Int. J. Rock Mechanics and Mining Sciences, vol. 37, pp. 63-78,2000.

7. N. Khalili and A.P.S. Selvadurai, A Fully Coupled Constitutive Model for Thermohydro-mechanical Analysis in Elastic Media with Double Porosity, Geophys. Res. Lett., vol. 30, Art. No. 2268, 2003.

8. S.R. Pride and J.G. Berryman, Linear Dynamics of Double-porosity Dual-permeability Materials-I. Phys. Rev. vol.E 68, $036603,2003$.

9. Y. Zhao and M. Chen, Fully Coupled Dual-porosity Model for Anisotropic Formations. Int. J. Rock Mech. Min. Sci., vol. 43, pp. 1128-1133, 2006.

10. M. Svanadze, Dynamical Problems of the Theory of Elasticity for Solids with Double Porosity, Proceedings in Applied Mathematics and Mechanics, vol.10, pp. 309-310, 2010.

11. A. Ainouz, Homogenized double porosity models for poro-elastic media with interfacial flow barrier, Mathematica Bohemica, vol. 136, pp. 357-365, 2011.

12. M. Svanadze, Plane waves and boundary value problems in the theory of elasticity for solids with double porosity. Acta Applicandae Mathematicae, vol.122, pp.461471, 2012.

13. B. Straughan, Stability and Uniqueness in Double Porosity Elasticity, Int.J.Eng. Sci., vol.65, pp.1-8, 2013.

14. S.C. Cowin, Bone Poroelasticity, J. Biomech., vol. 32, pp. 217-238, 1999.

15. J. W. Nunziato and S.C. Cowin, A Nonlinear Theory of Elastic Materials With Voids, Arch. Rat. Mech. Anal., vol. 72, pp. 175-201, 1979.

16. S. C. Cowin and J.W. Nunziato, Linear Elastic Materials with Voids, J. Elasticity, vol.13, pp. 125-147, 1983.

17. S. C. Cowin and P. Puri, The Classical Pressure Vessel Problems for Linear Elastic Materials with Voids, J. Elasticity, vol. 13, pp.157-163,1983.

18. A. Bedford and D. S. Drumheller, Recent Advances: Theories of Immiscible and Structured Mixtures, Int. J. Eng. Sci., vol. 21, pp. 863-960, 1983.

19. D. Iesan, Thermoelastic Models of Continua. Kluwer Academic, Dordrecht,2004.

20. M. Ciarletta and B. Straughan, Thermo-poroacoustic Acceleration Waves in Elastic Materials with Voids, J. Math. Anal. Appl., vol.333, pp.142-150, 2007. 
21. A. Magaña and R. Quintanilla, On the Decay of in Porous-elasticity with Quasistatic Microvoids, J. Math.Anal. Appl. ,vol.331, pp. 617-630, 2007.

22. J. E. Muñoz-Rivera and R. Quintanilla, On the Time Polynomial Decay in Elastic Solids with Voids, J Math. Anal. Appl., vol.338, pp.1296- 1309, 2008.

23. P. X. Pamplona, J.E. Muñoz-Rivera and R. Quintanilla, Stabilization in Elastic Solids with Voids, J. Math. Anal. Appl., vol.350, pp. 37-49, 2009.

24. D. Iesan and R. Quintanilla, On Thermoelastic Bodies with Inner Structure and Microtemperatures, J. Math. Anal. Appl., vol.354, pp.12- 23, 2009.

25. M.C. Leseduarte, A. Magaña and R. Quintanilla, On the time Decay of Solutions in Porous-thermo-elasticity of Type II. Disc. Cont. Dyn. Syst. Series B, vol. 13 pp. 375-391, 2010.

26. P. X. Pamplona, J.E. Muñoz-Rivera and R. Quintanilla, Analyticity in Porousthermoelasticity with Microtemperatures.J. Math. Anal. Appl., vol.394, pp. 645$655,2012$.

27. A.E. Green and R.S. Rivlin, Multipolar Continuum Mechanics. Arch. Rational Mech. Anal., vol. 17, pp. 113-147, 1964.

28. Liu, Z., Zheng, S. (1999). Semigroups associated with dissipative systems, Chapman \& Hall/CRC Research Notes in Mathematics, vol. 398, Chapman \& Hall/CRC, Boca Raton, FL.

\section{APPENDIX}

The aim of the appendix is to prove that

$$
\begin{gathered}
\mu>0,3 \lambda+2 \mu>0, \quad \alpha_{2}>0, \quad \alpha_{1} \alpha_{2}-\alpha_{3}^{2}>0, \\
(3 \lambda+2 \mu)\left(\alpha_{1} \alpha_{2}-\alpha_{3}^{2}\right)>3\left(\alpha_{1} d^{2}+\alpha_{2} b^{2}-2 \alpha_{3} b d\right), \quad \alpha>0, \quad \alpha \gamma>b_{1}^{2} .
\end{gathered}
$$

is a family of necessary and sufficient conditions to guarantee that the internal energy is positive definite in the isotropic and homogeneous case.

It is clear that a necessary and sufficient condition is that

$$
\mu>0, \alpha>0, \alpha \gamma>b_{1}^{2}
$$

and that the matrix

$$
\left(\begin{array}{ccccc}
\lambda+2 \mu & \lambda & \lambda & b & d \\
\lambda & \lambda+2 \mu & \lambda & b & d \\
\lambda & \lambda & \lambda+2 \mu & b & d \\
b & b & b & \alpha_{1} & \alpha_{3} \\
d & d & d & \alpha_{3} & \alpha_{2}
\end{array}\right)
$$


is positive definite. If we apply the Sylvester rule we see that this matrix is positive definite if and only if

$$
\begin{gathered}
3 \lambda+2 \mu>0,(3 \lambda+2 \mu) \alpha_{1}>3 b^{2}, \\
(3 \lambda+2 \mu)\left(\alpha_{1} \alpha_{2}-\alpha_{3}^{2}\right)>3\left(\alpha_{1} d^{2}+\alpha_{2} b^{2}-2 \alpha_{3} b d\right) .
\end{gathered}
$$

We can write our bilinear form in an alternative order to consider the matrix

$$
\left(\begin{array}{ccccc}
\alpha_{2} & \alpha_{3} & d & d & d \\
\alpha_{3} & \alpha_{1} & b & b & b \\
d & b & \lambda+2 \mu & \lambda & \lambda \\
d & b & \lambda & \lambda+2 \mu & \lambda \\
d & b & \lambda & \lambda & \lambda+2 \mu
\end{array}\right) .
$$

In this case we obtain that a family of conditions is (taking in mind that $\mu>0$ )

$$
\begin{gathered}
\alpha_{2}>0, \alpha_{1} \alpha_{2}-\alpha_{3}^{2}>0 \\
(3 \lambda+2 \mu)\left(\alpha_{1} \alpha_{2}-\alpha_{3}^{2}\right)>3\left(\alpha_{1} d^{2}+\alpha_{2} b^{2}-2 \alpha_{3} b d\right) .
\end{gathered}
$$

Therefore (100)-(102) is equivalent to (100), (103), (104). First we see in a clear way that (100)-(102) implies (98), (99). On the other side we have to see from (98), (99) that

$$
(3 \lambda+2 \mu) \alpha_{1}>3 b^{2},
$$

but this is a direct consequence of the equivalence between the two family of conditions (100)-(102) and (100), (103), (104). 